Algebraic $\&$ Geometric $\mathcal{T}_{\text {opology }}$

Volume 5 (2005) 691-711

Published: 5 July 2005

ATG

\title{
Some analogs of Zariski's Theorem on nodal line arrangements
}

\author{
A.D.R. ChOUdARY \\ A. DIMCA \\ Ş. PAPADIMA
}

\begin{abstract}
For line arrangements in $\mathbb{P}^{2}$ with nice combinatorics (in particular, for those which are nodal away the line at infinity), we prove that the combinatorics contains the same information as the fundamental group together with the meridianal basis of the abelianization. We consider higher dimensional analogs of the above situation. For these analogs, we give purely combinatorial complete descriptions of the following topological invariants (over an arbitrary field): the twisted homology of the complement, with arbitrary rank one coefficients; the homology of the associated Milnor fiber and Alexander cover, including monodromy actions; the coinvariants of the first higher non-trivial homotopy group of the Alexander cover, with the induced monodromy action.
\end{abstract}

AMS Classification 32S22, 55N25; 14F35, 52C35, 55Q52

Keywords Hyperplane arrangement, oriented topological type, 1-marked group, intersection lattice, local system, Milnor fiber, Alexander cover

\section{Introduction}

Let $\mathcal{A}=\left\{H_{0}, \ldots, H_{n}\right\}$ be a line arrangement in the complex projective plane $\mathbb{P}^{2}$ with complement $M=M(\mathcal{A})=\mathbb{P}^{2} \backslash \cup_{i=0}^{n} H_{i}$, and fundamental group $\pi=$ $\pi_{1}(M)$. One has the following known result.

Theorem 1.1 The following statements are equivalent.

(i) $\mathcal{A}$ has only double points.

(ii) The fundamental group $\pi$ is abelian.

The implication (i) $\Longrightarrow$ (ii) goes back to Zariski [40, Chapter VIII, section 2 (see especially his comments on the proof of Theorem 1) and to his attempt to 
use it in the proof of his conjecture on the commutativity of the fundamental group of the complement of any nodal curve. See Deligne [5] and Fulton [14] for a proof of this conjecture, and Dimca [6, Corollary (4.3.18)] for details on Zariski's approach. Concerning this implication, note that Hattori [15] proves even more, namely that the complement $M$ is homotopy equivalent to the 2skeleton of the standard $n$-dimensional real torus $T_{n}$.

The implication (ii) $\Longrightarrow$ (i) is easy. Assume that $\mathcal{A}$ has a point $p$ of multiplicity $k>2$ and let $\mathcal{B}$ be the subarrangement of $\mathcal{A}$ consisting of all the lines passing through $p$. Then the inclusion $M(\mathcal{A}) \rightarrow M(\mathcal{B})$ induces an epimorphism at the level of fundamental groups and $\pi_{1}(M(\mathcal{B}))$ is a free group $\mathbb{F}_{k-1}$ on $k-1$ generators. Indeed, one has $M(\mathcal{B})=(\mathbb{C} \backslash\{k-1$ points $\}) \times \mathbb{C}$, hence $\pi_{1}(M(\mathcal{B}))=\mathbb{F}_{k-1}$. This implies that $\pi=\pi_{1}(M(\mathcal{A}))$ is not abelian, actually, not even nilpotent.

Note also that the implication (ii) $\Longrightarrow$ (i) is specific to line arrangements. In fact, there are many non-nodal curves $C \subset \mathbb{P}^{2}$ with $\pi_{1}\left(\mathbb{P}^{2} \backslash C\right)$ commutative, see for such examples [6, (4.3.8)].

In the first part of this paper we investigate to what extent the above Theorem 1.1 still holds when we consider affine nodal arrangements in the plane $\mathbb{C}^{2}$. In other words, we assume that $H_{0}$ is the line at infinity $L_{\infty}=\mathbb{P}^{2} \backslash \mathbb{C}^{2}$ and we ask that $\mathcal{A}$ has only double points in the affine plane $\mathbb{C}^{2}$. If there is just one multiple point on the line at infinity (i.e. when all the lines in $\mathcal{A}$ belong to the same pencil of lines in $\mathbb{P}^{2}$ ), then $M(\mathcal{A})$ is of a very special type, namely a product $(\mathbb{C} \backslash\{n$ points $\}) \times \mathbb{C}$, which is very easy to treat as an exercise.

In the sequel we assume that there are exactly $r \geq 2$ multiple points on the line at infinity $H_{0}$, and that they are of multiplicities $m_{1}+1, \ldots, m_{r}+1$. In this situation we say that $\mathcal{A}$ is split solvable of type $\mathbf{m}=\left(m_{1}, m_{2}, \ldots, m_{r}\right)$. The arrangements having this simple type of combinatorics belong to the hypersolvable class introduced by Jambu and Papadima, see [18, p.1142]. The type $\mathbf{m}$ determines the combinatorics of the arrangement $\mathcal{A}$, i.e. its intersection lattice $L(\mathcal{A})$, defined as in [30]. Here is a simple way to think about the type $\mathbf{m}$ : there are exactly $r$ directions for the lines in the affine arrangement in $\mathbb{C}^{2}$ and there are exactly $m_{j}$ parallel lines having the $j$-th direction as their common direction, for any $j=1, \ldots, r$. Requiring only double intersection points, for the $m_{1}+\ldots+m_{r}$ affine lines in $\mathbb{C}^{2}$, determines then the lattice.

The ideal generalization of Theorem 1.1] would be that the following data are equivalent (i.e. they determine each other):

(I) the combinatorics of $\mathcal{A}$ as described by $\mathbf{m}$; 
(II) the fundamental group $\pi=\pi_{1}(M(\mathcal{A}))$.

Such a result would be quite interesting, since in general it is known that the combinatorics does not determine the fundamental group of a line arrangement, see Rybnikov [36. However, such a straightforward generalization is not true. Indeed, M. Falk has produced in [12, p.146-147] an example of two line arrangements $\mathcal{A}_{1}$ and $\mathcal{A}_{2}$ such that $\mathcal{A}_{1}$ is split solvable of type $(1,2,2), M\left(\mathcal{A}_{1}\right)$ is homotopy equivalent to $M\left(\mathcal{A}_{2}\right)$, but $\mathcal{A}_{1}$ and $\mathcal{A}_{2}$ are combinatorially distinct.

To state our first result we need some definitions. We assume from now on that all arrangements are ordered, i.e. they come with a numbering of their hyperplanes. All complex varieties are oriented using the corresponding complex orientations.

The first definition was systematically investigated by Papadima in [32, in analogy with classical knot theory.

Definition 1.2 Two arrangements $\mathcal{A}=\left\{H_{0}, \ldots, H_{n}\right\}$ and $\mathcal{B}=\left\{\bar{H}_{0}, \ldots, \bar{H}_{n}\right\}$ in the same ambient projective space $\mathbb{P}^{N}$ are said to have the same oriented topological type (notation $\mathcal{A} \approx \mathcal{B}$ ) if there is an orientation-preserving homeomorphism $f: \mathbb{P}^{N} \rightarrow \mathbb{P}^{N}$, inducing for all $k=0, \ldots, n$ orientation-preserving homeomorphisms $f \mid H_{k}: H_{k} \rightarrow \bar{H}_{k}$.

The second definition we need is the following.

Definition 1.3 Let $G$ be a group whose abelianization $G_{a b}$ is free abelian of finite rank. Then a 1-marking of $G$ is a choice of an ordered basis for $G_{a b}$. If $G$ and $G^{\prime}$ are two 1-marked groups, we say that $G$ and $G^{\prime}$ are 1-marked isomorphic if there is a group isomorphism $\phi: G \rightarrow G^{\prime}$ preserving in the obvious sense the two markings. Notation: $G \approx_{1} G^{\prime}$.

Example 1.4 If $G=\pi=\pi_{1}(M(\mathcal{A}))$ is the fundamental group of an ordered projective arrangement $\mathcal{A}=\left\{H_{0}, \ldots, H_{n}\right\}$, then the meridians $\left\{x_{1}, \ldots, x_{n}\right\}$ give a geometric 1-marking for $G_{a b}=H_{1}(M(\mathcal{A}), \mathbb{Z})$. Here $x_{i}$ is the homology class of a small loop $\gamma_{i}$ going in the positive direction around the hyperplane $H_{i}$. The fundamental group of an ordered projective arrangement is always in this paper endowed with this geometric 1-marking.

Moreover, it is known that $\mathcal{A} \approx \mathcal{B}$ implies $\pi_{1}(M(\mathcal{A})) \approx_{1} \pi_{1}(M(\mathcal{B}))$ with $\phi=f_{\sharp}$; see Lemma 2.7 from 32 . 
Remark 1.5 If $G$ and $G^{\prime}$ are two finitely presented groups, there is no algorithm to decide whether they are or not isomorphic. However, if $G$ and $G^{\prime}$ have in addition only commutator relations (which is the case for $\pi_{1}(M(\mathcal{A}))$ ), then there is an algorithmic obstruction theory for an analogous 1,2-marked isomorphism problem, see Papadima [32, Section 4]. Moreover, this reduces to the above $\approx_{1}$-problem, when $G$ and $G^{\prime}$ are fundamental groups of projective arrangements with the same combinatorics, see [32, Remark 4.7]. The second obstruction in this sense plays a key role in Rybnikov's work [36].

Now we can state our first result. For a type $\mathbf{m}=\left(m_{1}, m_{2}, \ldots, m_{r}\right)$, consider the projective space $\mathbb{P}^{r}$ with coordinates $z_{0}, z_{1}, \ldots, z_{r}$ and the arrangement $\widehat{\mathcal{A}}(\mathbf{m})$ therein given by

$z_{0}\left(z_{1}-z_{0}\right)\left(z_{1}-2 z_{0}\right) \cdots\left(z_{1}-m_{1} z_{0}\right)\left(z_{2}-z_{0}\right) \cdots\left(z_{2}-m_{2} z_{0}\right) \cdots\left(z_{r}-m_{r} z_{0}\right)=0$.

Here the hyperplanes in $\widehat{\mathcal{A}}(\mathbf{m})$ inherit the order of the corresponding linear factors in the above product. We set $\mathcal{A}(\mathbf{m})=\widehat{\mathcal{A}}(\mathbf{m}) \cap U$, where $U$ is a general 2-plane in $\mathbb{P}^{r}$, which we identify to $\mathbb{P}^{2}$. It is obvious that the arrangement $\mathcal{A}(\mathbf{m})$ is split solvable of type $\mathbf{m}$ and that $M(\widehat{\mathcal{A}}(\mathbf{m}))$ is isomorphic to the product

$$
\left(\mathbb{C} \backslash\left\{m_{1} \text { points }\right\}\right) \times \cdots \times\left(\mathbb{C} \backslash\left\{m_{r} \text { points }\right\}\right) .
$$

In particular, $\widehat{\mathcal{A}}(\mathbf{m})$ is aspherical. Note also that the combinatorics of $\mathcal{A}(\mathbf{m})$ is nice, in the sense of Jiang-Yau [20. Definition 3.2]; see $\S 2.7$

It turns out that the appropriate generalization of Theorem 1.1 actually holds for an arbitrary nice combinatorics.

Theorem 1.6 Let $\mathcal{A}$ and $\mathcal{B}$ be line arrangements in $\mathbb{P}^{2}$, with $\mathcal{B}$ nice. The following are equivalent.

(i) $L(\mathcal{A})=L(\mathcal{B})$ (i.e. they have the same combinatorics);

(ii) $\mathcal{A} \approx \mathcal{B}$ (i.e. they have the same oriented topological type);

(iii) $M(\mathcal{A}) \sim M(\mathcal{B})$ (i.e. their complements are homeomorphic);

(iv) $\pi_{1}(M(\mathcal{A})) \approx_{1} \pi_{1}(M(\mathcal{B}))$ (i.e. their fundamental groups are 1-marked isomorphic).

The implication (iv) $\Rightarrow$ (i), which corresponds to (ii) $\Rightarrow$ (i) in Zariski's classical set-up from Theorem 1.1 will be proved in the next section for arbitrary line arrangements. In fact this will appear as a special case of a result on general hyperplane arrangements, which identifies precisely the amount of combinatorics determined by the 1-marked fundamental group of the complement, see Theorem 2.1. The other implications follow from results obtained by Jiang-Yau in [19, 20. Theorem 1.6] has the following consequence. 
Corollary 1.7 Let $\mathcal{A}$ be a line arrangement in $\mathbb{P}^{2}$. The following are equivalent.

(i) $\mathcal{A}$ has the same combinatorics as a split solvable arrangement of type $\mathbf{m}=\left(m_{1}, m_{2}, \ldots, m_{r}\right)$;

(ii) $\pi_{1}(M(\mathcal{A})) \approx_{1} \mathbb{F}(\mathbf{m})$, where the product of free groups, $\mathbb{F}(\mathbf{m})=\mathbb{F}_{m_{1}} \times$ $\cdots \times \mathbb{F}_{m_{r}}$, is endowed with the obvious 1-marking;

(iii) $\mathcal{A} \approx \mathcal{A}(\mathbf{m})$.

Following Zariski's ideas, Oka and Sakamoto [28] gave a general condition for the splitting of the fundamental group of the complement of a projective plane curve as a direct product. This was used by Fan [13] to derive a sufficient condition (a priori not combinatorial, but verified by the split solvable class) under which the fundamental group of a line arrangement is a product of free groups. However, Fan's paper leaves the converse implication as an open question, see [13, p.290].

An important consequence of Zariski's conjecture is that the fundamental group of a nodal curve has a simple explicit description, involving only the degree list of the irreducible components of the curve, see for instance [6]. This viewpoint is pursued in the second part of our paper. More precisely, we extend the implication (i) $\Longrightarrow$ (ii) from Corollary 1.7 above to higher dimensional hyperplane arrangements with simple combinatorics of split solvable type. We show that not only the fundamental group, but also a lot of additional important topological information on the complement can be explicitly described in combinatorial terms. Such computations do not seem at all obvious, for an arbitrary nice combinatorics.

Set $M(\mathbf{m}, s):=M(\widehat{\mathcal{A}}(\mathbf{m})) \cap U^{s}$, where $U^{s} \subset \mathbb{P}^{r}$ is a generic $s$-plane, for $2 \leq s \leq$ $r$. Note that $M(\mathbf{m}, 2)=M(\mathcal{A}(\mathbf{m}))$, and $M(\mathbf{m}, r)=M(\widehat{\mathcal{A}}(\mathbf{m}))=K(\mathbb{F}(\mathbf{m}), 1)$. We will examine four topological invariants of $M(\mathbf{m}, s)$, in sections 3 and 4 , using tools from [10, [1].

In Section 3, we will look at twisted homology with rank one coefficients, and Milnor fiber homology. Both topics are under current intense investigation in arrangement theory. The important role played in singularity theory by the homology of the Milnor fiber of a polynomial, together with the monodromy action on it, can be traced back to Milnor's seminal book [27]. For the importance of twisted rank one homology in arrangement theory, and connexions with hypergeometric integrals, see the book by Orlik-Terao [31.

The rank one local systems $\mathcal{L}_{\rho}$ on $M(\mathbf{m}, s)$ are parametrized by group homomorphisms, $\rho: \mathbb{F}(\mathbf{m}) \rightarrow \mathbb{K}^{*}$, where $\mathbb{K}$ is an arbitrary field. The computation of 
all Betti numbers of $M(\mathbf{m}, s)$ with coefficients in $\mathcal{L}_{\rho}$ is carried out in Proposition [3.4. The result is purely combinatorial, in the sense that it depends only on $\mathbf{m}, s$, and on the subset of those indices $i$ for which the restriction of $\rho$ to $\mathbb{F}_{m_{i}}$ is non-trivial.

The Milnor fiber $F:=f^{-1}(1)$ of a homogeneous degree $d$ polynomial mapping, $f: \mathbb{C}^{s+1} \rightarrow \mathbb{C}$, is endowed with a geometric monodromy, induced by the diagonal action of the cyclic group of complex $d$-roots of unity on $\mathbb{C}^{s+1}$. Denote by $F(\mathbf{m}, s)$ the Milnor fiber of the defining polynomial of the cone over the arrangement $\widehat{\mathcal{A}}(\mathbf{m}) \cap U^{s}$. Our main result in Section 3 is Theorem 3.7 where we give a complete description of $H_{*}(F(\mathbf{m}, s), \mathbb{K})$, for an arbitrary field $\mathbb{K}$, together with the $\mathbb{K}_{\mathbb{Z}}$-module structure induced by the geometric monodromy action. Since the result depends only on $\mathbf{m}$ and $s$, we deduce in particular that $H_{*}(F(\mathbf{m}, s), \mathbb{Z})$ is torsion-free. This is to be compared to the examples found by Cohen-Denham-Suciu in [2], where multiarrangements (i.e. arrangements defined by non-reduced equations) with torsion in $H_{1}(F, \mathbb{Z})$ are constructed. Note also that the torsion-freeness of $H_{1}(F, \mathbb{Z})$ in the arrangement (i.e. reduced) case is still a major open question.

In Section 4, we examine the Alexander cover, $E(\mathbf{m}, s) \rightarrow M(\mathbf{m}, s)$. This is the $\mathbb{Z}$-cover of $M(\mathbf{m}, s)$ with fundamental group $N(\mathbf{m}):=\operatorname{ker}\left(\nu: \pi_{1}(M(\mathbf{m}, s))=\right.$ $\mathbb{F}(\mathbf{m}) \rightarrow \mathbb{Z})$, where $\nu$ sends to 1 all generators of $\mathbb{F}(\mathbf{m})$. This definition mimicks a classical construction from the theory of Alexander invariants of knots and links in $S^{3}$, see for instance Hillman's book 16. In singularity theory, Alexander invariants were introduced and studied by Libgober, in a series of papers starting with [22], see also [23], 24], 25], 26] as well as [9].

Our first main result in Section 4 is Theorem 4.2, where we give a complete description of the graded $\mathbb{K} \mathbb{Z}$-module $H_{*}(E(\mathbf{m}, s), \mathbb{K})$ over an arbitrary field $\mathbb{K}$, only in terms of $\mathbf{m}$ and $s$.

Our second main result here is related to higher homotopy groups of Alexander covers. Obviously, $E(\mathbf{m}, r)=K(N(\mathbf{m}), 1)$ is aspherical. Assuming $2 \leq s<r$, it turns out that the first higher non-trivial homotopy group of $E(\mathbf{m}, s)$ is $\pi_{s} E(\mathbf{m}, s)$. We give a concrete estimate of its non-triviality, in the following way. Let $\mathbb{K}$ be an arbitrary field. As is well-known, the $N(\mathbf{m})$-coinvariants,

$$
\left(\pi_{s} E(\mathbf{m}, s) \otimes \mathbb{K}\right)_{N(\mathbf{m})},
$$

have a natural $\mathbb{K} \mathbb{Z}$-module structure. We describe it completely, in Theorem 4.4 again solely in terms of $\mathbf{m}$ and $s$. Finally, we note in Remark 4.5, as a result of the aforementioned computation, that $\pi_{s} E(\mathbf{m}, s)$ is not finitely generated over $\mathbb{Z} N(\mathbf{m})$, in spite of the fact that additively

$$
\pi_{s} E(\mathbf{m}, s)=\pi_{s} M(\mathbf{m}, s),
$$


and the latter is known to have a finite free resolution over $\mathbb{Z F}(\mathbf{m})$, see [10].

Remark 1.8 The statements (i) and (ii) in Theorem 1.1 are equivalent to the following.

(iii) The second Betti number of $M$ is maximal in the set of second Betti numbers of complements of arrangements of $n+1$ lines in $\mathbb{P}^{2}$. Moreover this maximum is given by $b_{2}(M)=\left(\begin{array}{l}n \\ 2\end{array}\right)$.

The equivalence of (i) and (iii) follows from the description of the cohomology of the complement of the associated central arrangement $\mathcal{A}^{\prime}$ in $\mathbb{C}^{3}$ given by Orlik and Solomon in 29, since the non-nodal points $p$ correspond exactly to nontrivial relations in $H^{2}\left(M\left(\mathcal{A}^{\prime}\right)\right)$. A further generalization to curve arrangements will be given elsewhere.

\section{Combinatorics and 1-marked groups}

This section is devoted to the proof of Theorem 1.6 and Corollary 1.7 Our key result in this direction is the following.

Theorem 2.1 Let $\mathcal{A}$ and $\mathcal{B}$ be arbitrary projective arrangements. Assume that $\pi_{1}(M(\mathcal{A})) \approx_{1} \pi_{1}(M(\mathcal{B}))$. Set $p:=\min \left\{p_{\mathcal{A}}, p_{\mathcal{B}}\right\}$. Then $L_{p}(\mathcal{A})=L_{p}(\mathcal{B})$. In particular $L_{2}(\mathcal{A})=L_{2}(\mathcal{B})$.

Here $p_{\mathcal{A}}$ stands for the order of $\pi_{1}$-connectivity $p(M(\mathcal{A}))$ of the complement $M(\mathcal{A})$, a homotopy invariant introduced by Papadima and Suciu in [33, p.73]. The notation $L_{p}(\mathcal{A})=L_{p}(\mathcal{B})$ means that $\mathcal{A}$ and $\mathcal{B}$ have the same dependent subarrangements of cardinality at most $p+1$.

Example 2.2 For line arrangements, the above result simply says that the 1-marked group $\pi_{1}(M(\mathcal{A}))$ determines the intersection lattice $L(\mathcal{A})$. It seems worth mentioning that this phenomenon may also happen in higher dimensions. Indeed, let $\widehat{\mathcal{A}}$ and $\widehat{\mathcal{B}}$ be essential aspherical arrangements in $\mathbb{P}^{s}$ and $\mathbb{P}^{t}$ respectively. Take $(r-1)$-generic proper sections $\mathcal{A}:=\widehat{\mathcal{A}} \cap U$ and $\mathcal{B}:=\widehat{\mathcal{B}} \cap V$, with $r>2$. Then $p_{\mathcal{A}}=p_{\mathcal{B}}=r-1$ by [10, Theorem 18(i)], in particular $\mathcal{A}$ and $\mathcal{B}$ are non-aspherical. Since $\operatorname{rank}(\mathcal{A})=\operatorname{rank}(\mathcal{B})=r$, we deduce from Theorem 2.1 that $\pi_{1}(M(\mathcal{A})) \approx_{1} \pi_{1}(M(\mathcal{B}))$ implies $L(\mathcal{A})=L(\mathcal{B})$. 


\subsection{Combinatorics and 1-marked cohomology}

To prove Theorem 2.1 we begin by recalling that the cohomology algebra of the complement, together with its natural 1-marking, contains the same information as the combinatorics. More precisely, we will need the result below, essentially stated (without proof) by Kawahara in [21, Lemmas 22 and 23]. For the reader's convenience, we include a short proof.

Proposition 2.4 Let $\mathcal{A}^{\prime}=\left\{H_{0}^{\prime}, \ldots, H_{n}^{\prime}\right\}$ be a central arrangement in $\mathbb{C}^{N}$. For $i=0,1, \ldots, n$, let $e_{i} \in H^{1}\left(M\left(\mathcal{A}^{\prime}\right)\right)$ be the cohomology class corresponding to the hyperplane $H_{i}^{\prime}$. Then the cohomology algebra $H^{*}\left(M\left(\mathcal{A}^{\prime}\right)\right)$ and the collection of elements $\left\{e_{0}, \ldots, e_{n}\right\}$ determine the combinatorics of $\mathcal{A}^{\prime}$.

Proof It is enough to notice that, for any multi-index $i_{1}<\ldots<i_{p}$, one has

$$
\operatorname{codim}\left(H_{i_{1}}^{\prime} \cap \ldots \cap H_{i_{p}}^{\prime}\right)<p
$$

if and only if

$$
\sum_{j=1, p}(-1)^{j} e_{i_{1}} \wedge \ldots \wedge \hat{e}_{i_{j}} \wedge \ldots \wedge e_{i_{p}}=0
$$

in $H^{p-1}\left(M\left(\mathcal{A}^{\prime}\right)\right)$.

Indeed, the "only if" part follows from the description of the cohomology algebra in [29], while the "if" part follows by applying Proposition 3.66 in 30 to the subarrangement of $\mathcal{A}^{\prime}$ consisting of the hyperplanes $\left\{H_{i_{1}}^{\prime}, \ldots, H_{i_{p}}^{\prime}\right\}$.

\subsection{More on 1-markings}

As indicated by the previous result, it will be useful to describe the general relationship between various types of 1 -markings.

Let $\mathcal{A} \subset \mathbb{P}^{N}$ be an arrangement and let $\mathcal{A}^{\prime} \subset \mathbb{C}^{N+1}$ be the associated central arrangement. Then $\pi^{\prime}=\pi_{1}\left(M\left(\mathcal{A}^{\prime}\right)\right)$ has a natural 1-marking $\left\{x_{0}^{\prime}, \ldots, x_{n}^{\prime}\right\}$, where $x_{i}^{\prime} \in H_{1}\left(\left(M\left(\mathcal{A}^{\prime}\right)\right)\right.$ is the homology class of a small loop $\gamma_{i}^{\prime}$ going in the positive direction around the affine hyperplane $H_{i}^{\prime}$ corresponding to the projective hyperplane $H_{i}$.

From the natural product decomposition, $M\left(\mathcal{A}^{\prime}\right)=M(\mathcal{A}) \times \mathbb{C}^{*}$ one gets natural isomorphisms

$$
\iota(\mathcal{A})_{\sharp}: \pi_{1}\left(M\left(\mathcal{A}^{\prime}\right)\right) \stackrel{\sim}{\rightarrow} \pi_{1}(M(\mathcal{A})) \times \mathbb{Z} u_{0}
$$


and

$$
\iota(\mathcal{A})_{*}: H_{1}\left(M\left(\mathcal{A}^{\prime}\right)\right) \stackrel{\sim}{\rightarrow} H_{1}(M(\mathcal{A})) \oplus \mathbb{Z} u_{0}
$$

where $u_{0}$ is the standard generator of $\pi_{1}\left(\mathbb{C}^{*}\right)=H_{1}\left(\mathbb{C}^{*}\right) \simeq \mathbb{Z}$. The isomorphism $\iota(\mathcal{A})_{*}$ sends the classes $x_{i}^{\prime}$ to $x_{i}$ for all $i=1, \ldots, n$ and the sum $x_{0}^{\prime}+x_{1}^{\prime}+\ldots+x_{n}^{\prime}$ to $u_{0}$, see [7, p.209]. It follows that, if $\mathcal{A}$ and $\mathcal{B}$ are two projective arrangements and $\phi: \pi_{1}(M(\mathcal{A})) \rightarrow \pi_{1}(M(\mathcal{B}))$ is a marked isomorphism, then

$$
\theta=\iota(\mathcal{B})_{\sharp}^{-1} \circ(\phi \times \mathrm{id}) \circ \iota(\mathcal{A})_{\sharp}
$$

gives an isomorphism $\pi_{1}\left(M\left(\mathcal{A}^{\prime}\right)\right) \rightarrow \pi_{1}\left(M\left(\mathcal{B}^{\prime}\right)\right)$ preserving the geometric 1markings.

A 1-marking $\left\{x_{1}, \ldots, x_{n}\right\}$ for a group $G$ induces, for any ring $R$, an ordered $R$-basis $\left\{x_{1}^{*}, \ldots, x_{n}^{*}\right\}$ for $H^{1}(G, R)=\operatorname{Hom}\left(H_{1}(G, \mathbb{Z}), R\right)$ given by the usual property, $x_{i}^{*}\left(x_{j}\right)=\delta_{i j}$, which will be called a cohomological 1-marking. If $\phi: G \rightarrow G^{\prime}$ is a 1-marked isomorphism, then $\phi^{*}: H^{1}\left(G^{\prime}, R\right) \rightarrow H^{1}(G, R)$ preserves the corresponding cohomological 1-markings.

\subsection{Proof of Theorem 2.1}

Let $\mathcal{A}^{\prime}$ and $\mathcal{B}^{\prime}$ be the associated central arrangements. Set $\pi_{\mathcal{A}}^{\prime}:=\pi_{1}\left(M\left(\mathcal{A}^{\prime}\right)\right)$, $K_{\mathcal{A}}^{\prime}:=K\left(\pi_{\mathcal{A}}^{\prime}, 1\right)$, denote by $f_{\mathcal{A}}: M\left(\mathcal{A}^{\prime}\right) \rightarrow K_{\mathcal{A}}^{\prime}$ the classifying map, and likewise for $\mathcal{B}$. Assuming that $\pi_{1}(M(\mathcal{A})) \approx_{1} \pi_{1}(M(\mathcal{B}))$, we get an algebra isomorphism $H^{*}\left(K_{\mathcal{A}}^{\prime}\right) \simeq H^{*}\left(K_{\mathcal{B}}^{\prime}\right)$, preserving the cohomological 1-markings, see subsection 2.5 .

We claim that $f_{\mathcal{A}}$ induces isomorphisms $f_{\mathcal{A}}^{q}: H^{q}\left(K_{\mathcal{A}}^{\prime}, \mathbb{Q}\right) \rightarrow H^{q}\left(M\left(\mathcal{A}^{\prime}\right), \mathbb{Q}\right)$, for all $q \leq p\left(M\left(\mathcal{A}^{\prime}\right)\right)=p(M(\mathcal{A}))$, and likewise for $\mathcal{B}$.

To check this, note first that $f_{\mathcal{A}}^{q}$ is surjective for any $q \geq 0$, since $f_{\mathcal{A}}^{1}$ is by construction an isomorphism and the algebra $H^{*}\left(M\left(\mathcal{A}^{\prime}\right), \mathbb{Q}\right)$ is generated by $H^{1}\left(M\left(\mathcal{A}^{\prime}\right), \mathbb{Q}\right),\left[29\right.$. Next, for $q \leq p_{\mathcal{A}}$, the vector spaces $H^{q}\left(K_{\mathcal{A}}^{\prime}, \mathbb{Q}\right)$ and $H^{q}\left(M\left(\mathcal{A}^{\prime}\right), \mathbb{Q}\right)$ have the same finite dimension, by the very definition of the order of $\pi_{1}$-connectivity $p_{\mathcal{A}}$, hence our claim. Note that $f_{\mathcal{A}}^{1}$ takes the cohomological 1-marking of $\pi_{\mathcal{A}}^{\prime}$ to the $H^{1}$-basis $\left\{e_{0}, \ldots, e_{n}\right\}$ appearing in Proposition 2.4 again by construction, and similarly for $f_{\mathcal{B}}^{1}$. We thus know that the cohomology algebras $H^{\leq} p\left(M\left(\mathcal{A}^{\prime}\right), \mathbb{Q}\right)$ and $H^{\leq p}\left(M\left(\mathcal{B}^{\prime}\right), \mathbb{Q}\right)$ are isomorphic, and the distinguished $H^{1}$-bases are preserved. By Proposition 2.4 we get $L_{p}(\mathcal{A})=L_{p}(\mathcal{B})$.

Finally, the order of $\pi_{1}$-connectivity $p_{\mathcal{A}}$ is at least 2 for any hyperplane arrange-

ment $\mathcal{A}$. This follows from a result of Randell [35], which says that $f_{\mathcal{A}}^{2}$ is always an isomorphism. The proof of Theorem 2.1 is complete. 


\subsection{Proof of Theorem 1.6}

Jiang and Yau first associate to an arbitrary line arrangement $\mathcal{A}$ a graph $\Gamma_{\mathcal{A}}$, depending only on $L(\mathcal{A})$, in the following way. The vertices $\mathcal{V}_{\mathcal{A}}$ are the singular points of $\mathcal{A}$ of multiplicity at least 3 . Two vertices are joined by an edge if there is a line of $\mathcal{A}$ containing them. For $v \in \mathcal{V}_{\mathcal{A}}$, they define a subgraph $\Gamma_{\mathcal{A}}(v)$, having as vertex set, $\mathcal{V}_{\mathcal{A}}(v), v$ and all his neighbours from $\Gamma_{\mathcal{A}}$. An edge $\left\{u, u^{\prime}\right\}$ belongs to $\Gamma_{\mathcal{A}}(v)$ if $u, u^{\prime}$ and $v$ lie on a line of $\mathcal{A}$. The following combinatorial definition appears in [20, Definition 3.2].

Definition 2.8 The arrangement $\mathcal{A}$ is nice if there is $\mathcal{V}^{\prime} \subset \mathcal{V}_{\mathcal{A}}$ such that $\mathcal{V}_{\mathcal{A}}\left(u^{\prime}\right) \cap \mathcal{V}_{\mathcal{A}}\left(v^{\prime}\right)=\emptyset$, for all distinct $u^{\prime}, v^{\prime} \in \mathcal{V}^{\prime}$, and with the property that the subgraph $\Gamma^{\prime} \subset \Gamma_{\mathcal{A}}$, obtained by deleting the vertex $v^{\prime}$ and the edges of $\Gamma_{\mathcal{A}}\left(v^{\prime}\right)$, for all $v^{\prime} \in \mathcal{V}^{\prime}$, is a forest.

If $\mathcal{A}$ is split solvable, then all vertices from $\mathcal{V}_{\mathcal{A}}$ lie on the line at infinity $H_{0}$ It follows that $\Gamma_{\mathcal{A}}$ is a complete graph, equal to $\Gamma_{\mathcal{A}}(v)$, for any $v \in \mathcal{V}_{\mathcal{A}}$. In particular $\Gamma^{\prime}$ is a discrete graph with no edges, and consequently $\mathcal{A}$ is nice.

We may now give the proofs of Theorem [1.6 and Corollary 1.7

(i) $\Longrightarrow$ (ii) If $L(\mathcal{A})=L(\mathcal{B})$, then $\mathcal{A}$ and $\mathcal{B}$ are lattice-isotopic; see 20, Theorem 3.3]. The conclusion follows from Randell's lattice-isotopy theorem 34.

(ii) $\Longrightarrow$ (iii) Obvious.

(iii) $\Longrightarrow$ (i) See [19].

(ii) $\Longrightarrow$ (iv) This implication is valid for arbitrary arrangements in $\mathbb{P}^{N}$, as noted in Example 1.4.

(iv) $\Longrightarrow$ (i) See our Theorem 2.1.

\subsection{Proof of Corollary 1.7}

To get Corollary 1.7 from Theorem 1.6, all we need is to prove the marked isomorphism $\pi_{1}(M(\mathcal{A}(\mathbf{m}))) \approx_{1} \mathbb{F}(\mathbf{m})$. This in turn follows from our definition of $\mathcal{A}(\mathbf{m})$ and from Zariski's Theorem on hyperplane sections, see for instance [6. p.26], saying that the inclusion $M(\mathcal{A}(\mathbf{m})) \rightarrow M(\widehat{\mathcal{A}}(\mathbf{m}))$ is a 2-equivalence. Therefore

$$
\pi_{1}(M(\mathcal{A}(\mathbf{m}))) \approx_{1} \pi_{1}(M(\widehat{\mathcal{A}}(\mathbf{m}))) \approx_{1} \mathbb{F}_{m_{1}} \times \cdots \times \mathbb{F}_{m_{r}}
$$




\section{Combinatorial formulae for twisted homology}

We know from Corollary 1.7 that the complement of a split-solvable line arrangement of type $\mathbf{m}, M(\mathcal{A})$, is homeomorphic to the combinatorial model, $M(\widehat{\mathcal{A}}(\mathbf{m})) \cap U^{2}$, where $U^{2} \subset \mathbb{P}^{r}$ is a generic 2-plane. Our next results will describe various topological invariants of $M(\mathcal{A})$, in terms of $\mathbf{m}$.

\subsection{The set-up}

It turns out that this may be done, at no extra cost, for all generic $s$-plane sections, $M(\mathbf{m}, s):=M(\widehat{\mathcal{A}}(\mathbf{m})) \cap U^{s}$, with $2 \leq s \leq r$. Set $M:=M(\mathbf{m}, s)$ and $\pi:=\pi_{1}(M)$. Theorem 18 from 10 guarantees the existence, up to homotopy, of a minimal CW-structure $Y$ on $M(\widehat{\mathcal{A}}(\mathbf{m}))=K(\mathbb{F}(\mathbf{m}), 1)$, whose $s$-skeleton is homotopy equivalent to $M: M \cong Y^{(s)}$.

We aim at computing twisted homology, $H_{*}(M, S)$, where $S$ is a (right) $\mathbb{Z} \pi-$ module. For this, we may use the $\pi$-equivariant chain complex of the universal cover of $Y$,

$$
C .(\widetilde{Y}): 0 \rightarrow \mathbb{Z} \pi \otimes C_{r} \rightarrow \cdots \rightarrow \mathbb{Z} \pi \otimes C_{s} \stackrel{d_{s}}{\rightarrow} \mathbb{Z} \pi \otimes C_{s-1} \rightarrow \cdots,
$$

where $C_{k}, 0 \leq k \leq r$, denotes the free abelian group generated by the $k$-cells of $Y$. Indeed, one knows (see e.g. [39, Chapter VI]) that

$$
H_{*}(M, S)=H_{*}\left(S \otimes_{\mathbb{Z} \pi} C_{\leq s}(\tilde{Y})\right) .
$$

Since $s \geq 2, \pi=\pi_{1}(M(\widehat{\mathcal{A}}(\mathbf{m}))$, which is a product of free groups, $\mathbb{F}(\mathbf{m})=$ $\mathbb{F}_{m_{1}} \times \cdots \times \mathbb{F}_{m_{r}}$. Consider now the well-known standard minimal resolution of $\mathbb{Z}$ over $\mathbb{Z F}\left(x_{1}, \ldots, x_{m}\right)$, where $\mathbb{F}\left(x_{1}, \ldots, x_{m}\right)$ is the free group on the letters $x_{1}, \ldots, x_{m}$, see for instance [17, pp.196-197]. Use then tensor products of resolutions, as in [17, p.222], to obtain a free resolution of $\mathbb{Z}$ over $\mathbb{Z} \pi$,

$$
C_{\bullet}(\pi): 0 \rightarrow \mathbb{Z} \pi \otimes C_{r} \rightarrow \cdots \rightarrow \mathbb{Z} \pi \otimes C_{s} \stackrel{\partial_{s}}{\rightarrow} \mathbb{Z} \pi \otimes C_{s-1} \rightarrow \cdots
$$

Note that both (3.1) and (3.3) are minimal free resolutions of $\mathbb{Z}$ over $\mathbb{Z} \pi$. Consequently, $C_{k}=\operatorname{Tor}_{k}^{\mathbb{Z} \pi}(\mathbb{Z}, \mathbb{Z})$, for all $k$. In more concrete terms, we see from the construction of resolution (3.3) that the graded abelian group $C_{\bullet}:=\oplus_{k=0}^{r} C_{k}$ equals

$$
C_{\bullet}=\bigotimes_{i=1}^{r} C_{\bullet}^{i}
$$

Here $C_{\bullet}^{i}$ is concentrated in degrees $\bullet=0$ and 1 , for all $i$, with graded pieces $\mathbb{Z}$ and $\mathbb{Z}^{m_{i}}$ respectively. Denoting by $\left\{v_{1}^{i}, \ldots, v_{m_{i}}^{i}\right\}$ the standard basis of $\mathbb{Z}^{m_{i}}$, 
and setting $\mathbb{F}_{m_{i}}=\mathbb{F}\left(x_{1}^{i}, \ldots, x_{m_{i}}^{i}\right)$, we may completely describe the resolution (3.3), as follows:

$$
\partial_{k}\left(1 \otimes v_{j_{1}}^{i_{1}} \otimes \cdots \otimes v_{j_{k}}^{i_{k}}\right)=\sum_{p=1}^{k}(-1)^{p-1}\left(x_{j_{p}}^{i_{p}}-1\right) \otimes v_{j_{1}}^{i_{1}} \otimes \cdots \widehat{v_{j_{p}}^{i_{p}}} \cdots \otimes v_{j_{k}}^{i_{k}} .
$$

We may now explain our computational strategy. For $S$ a principal ideal domain, with $\mathbb{Z} \pi$-module structure given by a change of rings, $\mathbb{Z} \pi \rightarrow S$, we may use [11. Lemma 2.10] to infer that the $S$-chain complexes $S \otimes_{\mathbb{Z} \pi} C_{\bullet}(\widetilde{Y})$ and $S \otimes_{\mathbb{Z} \pi} C_{\bullet}(\pi)$ are actually isomorphic, not just chain-homotopy equivalent. This will enable us to replace $C_{\leq s}(\widetilde{Y})$ in equation (3.2) by a simpler, explicit object, namely $C_{\leq s}(\pi)$ from (3.3).

One more remark will be useful. Each graded abelian group $C_{\bullet}^{i}$ appearing in (3.4) is actually a chain complex, with differential $\partial^{\prime}: \mathbb{Z}^{m_{i}} \rightarrow \mathbb{Z}$ given by

$$
\partial^{\prime}\left(v_{j}^{i}\right)=1, \quad \text { for } j=1, \ldots, m_{i} .
$$

We may thus view $C$ • as a tensor product chain complex,

$$
\left(C_{\bullet}, \partial^{\prime}\right)=\bigotimes_{i=1}^{r}\left(C_{\bullet}^{i}, \partial^{\prime}\right)
$$

\subsection{Local systems}

A rank one local system $\mathcal{L}_{\rho}$ of $\mathbb{K}$-vector spaces on $M=M(\mathbf{m}, s)$ corresponds to a representation $\rho: \mathbb{F}(\mathbf{m}) \rightarrow \mathbb{K}^{*}$, where $\rho=\left(\rho_{1}, \ldots, \rho_{r}\right)$ with $\rho_{i}: \mathbb{F}_{m_{i}} \rightarrow \mathbb{K}^{*}$ a representation of the free group $\mathbb{F}_{m_{i}}$. We will apply Theorem 4.5 (2) in 11 . to express the dimensions of the cohomology groups $H^{*}\left(M(\mathbf{m}, s), \mathcal{L}_{\rho}\right)$ in terms of the type $\mathbf{m}, s$ and the support of the representation

$$
\operatorname{supp}(\rho)=\left\{1 \leq i \leq r \mid \rho_{i} \neq 1\right\} .
$$

This extends the results of Hattori from [15, where the case $m_{1}=\ldots=m_{r}=1$ is treated.

For the above representation $\rho$ we define its Poincaré polynomial by the formula

$$
P_{\rho}(t)=\sum_{q \geq 0} b_{q}(\rho) t^{q}
$$

where

$$
b_{q}(\rho)=\operatorname{dim}_{\mathbb{K}} \operatorname{Tor}_{q}^{\mathbb{Z F}(\mathbf{m})}\left(\mathbb{Z}, \mathbb{K}_{\rho}\right) .
$$

To apply the aforementioned theorem, we will need the following standard computation. 
Lemma 3.3 Let $\rho: \mathbb{F}(\mathbf{m}) \rightarrow \mathbb{K}^{*}$ be an arbitrary representation. Then

$$
P_{\rho}(t)=\prod_{i \notin \operatorname{supp}(\rho)}\left(1+m_{i} t\right) \prod_{i \in \operatorname{supp}(\rho)}\left(m_{i}-1\right) t .
$$

Proof Firstly, one has the following equality.

$$
P_{\rho}(t)=\prod_{i=1, r} P_{\rho_{i}}(t) .
$$

This equality follows via the Künneth formula from resolution (3.3) or, alternatively, from the Künneth formula for cohomology with sheaf coefficients, see [7. p.117]. The case of a single free group is immediate, using the standard minimal resolution.

Denoting by $\sigma_{i}, 0 \leq i \leq r$, the value of the $i$-th elementary symmetric function on $\mathbf{m}=\left(m_{1}, \ldots, m_{r}\right)$, we may now state our next result.

Proposition 3.4 Lat $M(\mathbf{m}, s)$ denote the $s$-generic section of $M(\widehat{\mathcal{A}}(\mathbf{m}))$, for $2 \leq s \leq r$. Let $\rho: \pi_{1}(M(\mathbf{m}, s))=\mathbb{F}(\mathbf{m}) \rightarrow \mathbb{K}^{*}$ be an arbitrary rank one local system of $\mathbb{K}$-vector spaces on $M(\mathbf{m}, s)$. Then

$$
\operatorname{dim}_{\mathbb{K}} H_{j}\left(M(\mathbf{m}, s), \mathcal{L}_{\rho}\right)=\left\{\begin{array}{cl}
b_{j}(\rho), & \text { for } j<s \\
\sigma_{s}+\sum_{i=0}^{s-1}(-1)^{i+s}\left[\sigma_{i}-b_{i}(\rho)\right], & \text { for } j=s \\
0, & \text { for } j>s
\end{array}\right.
$$

where the twisted Betti numbers $\left\{b_{i}(\rho)\right\}_{0 \leq i \leq r}$ are computed in Lemma 3.3. In particular, for all $0 \leq j \leq s$, one has

$$
\operatorname{dim}_{\mathbb{K}} H_{j}(M(\mathbf{m}, s), \mathbb{K})=\sigma_{j}
$$

Proof As recalled in $M(\widehat{3.1}, \widehat{\mathcal{A}}(\mathbf{m})) \cong Y$ and $M(\mathbf{m}, s) \cong Y^{(s)}$, where $Y$ is a minimal complex. Obviously, the Poincaré polynomial of $\widehat{\mathcal{A}}(\mathbf{m})$ equals $\prod_{i=1, r}\left(1+m_{i} t\right)$. Therefore, $\chi(M(\mathbf{m}, s))=\sum_{j=0, s}(-1)^{j} \sigma_{j}$. Everything follows now from [11, Theorem $4.5(2)]$.

In the case of rank one twisted homology, where $S=\mathbb{K}$, things are easy, since we only need to perform computations involving the fundamental group, and we may finish by using an Euler characteristic argument. In the sequel, $S$ will be the $\mathbb{K}$-algebra of a cyclic group. To identify the $S$-module structure of

$$
H_{*}(M, S)=H_{*}\left(S \otimes_{\mathbb{Z} \pi} C_{\leq s}(\tilde{Y})\right)
$$

(see [39] Ch.VI]), we need more, namely to replace $S \otimes_{\mathbb{Z} \pi} C_{\bullet}(\tilde{Y})$ by $S \otimes_{\mathbb{Z} \pi} C_{\bullet}(\pi)$, as explained in 3.1 


\subsection{The homology of the associated Milnor fiber}

Let $\mathcal{A}^{\prime}$ be a central arrangement of $n+1$ hyperplanes in $\mathbb{C}^{N+1}$. Let $f=0$ be a reduced defining equation for $\mathcal{A}^{\prime}$ in $\mathbb{C}^{N+1}$. Then $f$ is a homogeneous polynomial of degree $d=n+1$. The affine smooth hypersurface $F$ given by the equation $f=1$ in $\mathbb{C}^{N+1}$ is called the associated Milnor fiber of the central arrangement $\mathcal{A}^{\prime}$. This space comes equipped with an order $d$ diffeomorphism $h$, the monodromy, given by $h(x)=\lambda \cdot x$, where $\lambda=\exp (2 \pi \sqrt{-1} / d)$. This diffeomorphism gives rise to an important (left) $R \mathbb{Z}_{d}$-module structure on $H_{*}(F, R)$, for any commutative ring $R$. Our next goal is to describe completely this structure, for the generic section $\mathcal{A}=\widehat{\mathcal{A}}(\mathbf{m}) \cap U^{s}$ and an arbitrary field $R=\mathbb{K}$, in terms of $\mathbf{m}$ and $s$.

Keeping the previous notation, we may do this as follows. Denote by $\nu: \pi \rightarrow \mathbb{Z}$ the (unique) abelian character which takes the value 1 on the distinguished basis of $\pi_{a b}=\mathbb{F}(\mathbf{m})_{a b}$. Note that $\mathbb{K Z}_{d}$ is a quotient ring of the principal ring $\mathbb{K} \mathbb{Z}$, with $\mathbb{Z} \pi$-module structure coming from the change of rings $\mathbb{Z} \pi \stackrel{\nu}{\rightarrow} \mathbb{K} \mathbb{Z} \rightarrow \mathbb{K} \mathbb{Z}_{d}$. One knows (see [4] and [7, (2.3.4)]) that $H_{*}(F, \mathbb{K})=H_{*}\left(M, \mathbb{K}_{d}\right)$, as $\mathbb{K}_{d^{-}}$ modules. It follows from 3.1 that

$$
H_{*}\left(M, \mathbb{K}_{d}\right)=H_{*}\left(\mathbb{K} \mathbb{Z}_{d} \otimes_{\mathbb{Z} \pi} C_{\leq s}(\pi)\right),
$$

as $\mathbb{K Z}_{d}$-modules.

We now remark that the differential of the chain complex $\mathbb{K}_{d} \otimes_{\mathbb{Z} \pi} C_{\bullet}(\pi)$ equals

$$
\left\{(\tau-1) \otimes \partial_{k}^{\prime}: \mathbb{K}_{d} \otimes C_{k} \rightarrow \mathbb{K} \mathbb{Z}_{d} \otimes C_{k-1}\right\}_{k},
$$

where $\partial^{\prime}$ is described in (3.7) and $\tau-1$ is the multiplication operator by $(\tau-1) \in \mathbb{K}_{d}, \tau$ being the canonical generator of $\mathbb{Z}_{d}$. This follows from (3.5), together with (3.6).

It follows from (3.11) that

$$
\operatorname{ker}\left((\tau-1) \otimes \partial_{k}^{\prime}\right)=\left(\mathbb{K} \mathbb{Z}_{d} \otimes \operatorname{ker}\left(\partial_{k}^{\prime} \otimes \mathbb{K}\right)\right) \bigoplus\left(\left(\mathbb{K} \mathbb{Z}_{d}\right)^{\mathbb{Z}} \otimes \operatorname{im}\left(\partial_{k}^{\prime} \otimes \mathbb{K}\right)\right) \text {, }
$$

where $\left(\mathbb{K}_{d}\right)^{\mathbb{Z}}:=\operatorname{ker}(\tau-1)=\mathbb{K}$ with trivial $\mathbb{K}_{d}-$ action, and

$$
\operatorname{im}\left((\tau-1) \otimes \partial_{k+1}^{\prime}\right)=(\tau-1) \mathbb{K} \mathbb{Z}_{d} \otimes \operatorname{im}\left(\partial_{k+1}^{\prime} \otimes \mathbb{K}\right) .
$$

We are thus led to compute the homology of $\left(C_{\bullet}, \partial^{\prime}\right)$.

Lemma 3.6 For any coefficient ring $R, H_{*}\left(C_{\bullet}, R\right)$ is concentrated in degree $*=r$, where it is free of rank $\left(m_{1}-1\right) \cdots\left(m_{r}-1\right)$.

Proof Follows from equations (3.6)-(3.7), via the Künneth formula. 
To state our main result in this section, we set

$$
z_{j}:=\sigma_{j}+\sum_{i=o}^{j-1}(-1)^{i+j} \sigma_{i}, \quad \text { for } \quad 0 \leq j \leq r .
$$

Theorem 3.7 Let $F(\mathbf{m}, s)$ denote the Milnor fiber of the generic $s$-section, $\widehat{\mathcal{A}}(\mathbf{m}) \cap U^{s}$. Set $d=1+\sigma_{1}$. Then

$$
H_{j}(F(\mathbf{m}, s), \mathbb{K})=\left\{\begin{array}{cl}
\mathbb{K}^{z_{j}+z_{j-1}}, & \text { for } j<s ; \\
\mathbb{K}^{z_{s-1} \oplus\left(\mathbb{K}_{d}\right)^{z_{s}},} & \text { for } j=s \\
0, & \text { for } j>s,
\end{array}\right.
$$

as $\mathbb{K} \mathbb{Z}_{d}$-modules, where $\mathbb{K}$ is an arbitrary field. In particular, $H_{*}(F(\mathbf{m}, s), \mathbb{Z})$ is torsion-free and the integral monodromy action is trivial in degrees $<s$.

Proof An Euler characteristic argument may be used to infer from Lemma 3.6 that $\operatorname{dim}_{\mathbb{K}} \operatorname{ker}\left(\partial_{j}^{\prime} \otimes \mathbb{K}\right)=z_{j}$, for $j=0, \ldots, r$. Recall that the $\mathbb{K} \mathbb{Z}_{d}$-module $H_{*}(F(\mathbf{m}, s), \mathbb{K})$ may be computed from (3.10). The first assertion of the theorem follows then from equations (3.11) - (3.13), together with Lemma 3.6. Since the $\mathbb{K}$-Betti numbers of $F(\mathbf{m}, s)$ are independent of $\mathbb{K}$, the $\mathbb{Z}$-homology has no torsion.

Remark 3.8 From (3.14) we see that $z_{r}=\left(m_{1}-1\right) \cdots\left(m_{r}-1\right)$ is positive, unless there is some $m_{i}$ equal to 1 . We note that always $z_{j}>0$, if $j<r$. This may be seen for instance as follows. If $z_{j}=0$, then $\partial_{j+1}^{\prime}=0$. On the other hand, plainly $C_{i} \neq 0$, for any $i \leq r$. Pick then any basis element $v \in C_{j+1}$ and compute $\partial_{j+1}^{\prime}(v)$ using (3.6)-(3.7) to obtain a contradiction.

Remark 3.9 The triviality of the monodromy action on $H^{1}(F(\mathbf{m}, 2), \mathbb{C})$ was obtained by the second author along different lines, using relations to the topology of a polynomial mapping $\mathbb{C}^{2} \rightarrow \mathbb{C}$, see [8, Corollary 3.3]. This was the starting point of our interest in the class of split solvable line arrangements, and their higher dimensional analogs.

The same triviality of the complex monodromy action holds in the dual case when a line arrangement $\mathcal{A}$ has a line along which the only singularities are nodes, see [26] and [3] for higher dimensional analogs of this result.

Note also that, for Milnor fibers associated to projective hypersurfaces, the triviality of the complex monodromy may occur even when the integral monodromy is not trivial, see [37. 


\section{Homology and homotopy groups of infinite cyclic covers}

Let $M$ be a connected complex. Any 1 -marking of $\pi:=\pi_{1}(M)$ gives rise to an abelian character, $\nu: \pi \rightarrow \mathbb{Z}$, which takes the value 1 on all elements of the distinguished basis of $\pi_{a b}$. The $\mathbb{Z}$-cover associated to $\nu, q: E \rightarrow M$, will be called the Alexander cover, by analogy with the classical Alexander polynomial theory (in one variable) for links. In this section, we will take $M=M(\mathbf{m}, s)$, like in Section 3, endowed with the canonical 1-marking of $\pi=\mathbb{F}(\mathbf{m})$, and compute two basic topological invariants of $E$.

\subsection{Homology of the Alexander cover}

The tools we have developed so far enable us to give the following complete description of the homology with field coefficients, which involves only $\mathbf{m}$ and $s$, as before.

Theorem 4.2 Denote by $E(\mathbf{m}, s), 2 \leq s \leq r$, the Alexander cover of the complement of the generic $s$-section of $\widehat{\mathcal{A}}(\mathbf{m})$. Then

$$
H_{j}(E(\mathbf{m}, s), \mathbb{K})=\left\{\begin{array}{cl}
\mathbb{K}^{z_{j}}, & \text { for } j<s \\
(\mathbb{K} \mathbb{Z})^{z_{s}}, & \text { for } j=s \\
0, & \text { for } j>s
\end{array}\right.
$$

as $\mathbb{K} \mathbb{Z}$-modules (where $\left\{z_{j}\right\}_{0 \leq j \leq r}$ are defined in (3.14)), for any field $\mathbb{K}$.

Proof It follows from [39, Ch.VI] that

$$
H_{*}(E, \mathbb{K})=H_{*}\left(\mathbb{K} \mathbb{Z} \otimes_{\mathbb{Z} \pi} C_{\leq s}(\tilde{Y})\right),
$$

as $\mathbb{K} \mathbb{Z}$-modules, where $C_{\bullet}(\tilde{Y})$ is as in (3.1) and the $\mathbb{Z} \pi$-module structure on $\mathbb{K} \mathbb{Z}$ is given by $\nu: \mathbb{Z} \pi \rightarrow \mathbb{K} \mathbb{Z}$. As explained in \$3.1, equation (4.1) above may be replaced by the simpler explicit form

$$
H_{*}(E, \mathbb{K})=H_{*}\left(\mathbb{K} \mathbb{Z} \otimes_{\mathbb{Z} \pi} C_{\leq s}(\pi)\right), \quad \text { over } \quad \mathbb{K} \mathbb{Z} .
$$

From now on, the computation is almost identical to the one from the proof of Theorem 3.7 from $\$ 3.5$, with $\mathbb{K} \mathbb{Z}_{d}$ replaced by $\mathbb{K} \mathbb{Z}$, except the fact that in the case of the Alexander cover we have $(\mathbb{K} \mathbb{Z})^{\mathbb{Z}}=\operatorname{ker}(\tau-1)=0$. 


\subsection{Homotopy of the Alexander cover}

Let us analyze now the homotopy groups of the Alexander cover, $q: E(\mathbf{m}, s) \rightarrow$ $M(\mathbf{m}, s)$. Set $N(\mathbf{m}):=\pi_{1}(E(\mathbf{m}, s))$. Clearly, $N(\mathbf{m})=\operatorname{ker}(\nu: \mathbb{F}(\mathbf{m}) \rightarrow \mathbb{Z})$. Since $M(\mathbf{m}, r)=M(\widehat{\mathcal{A}}(\mathbf{m}))$ is aspherical, $E(\mathbf{m}, r)=K(N(\mathbf{m}), 1)$. We will thus assume in the sequel that $2 \leq s \leq r-1$, and suppress $\mathbf{m}$ and $s$ from notation.

It follows from [10. Theorem 18(ii)] that $\pi_{s} E=\pi_{s} M$ is the first higher nontrivial homotopy group of $E$. Moreover, $\pi_{s} M$ has a finite free, minimal, $\mathbb{Z} \pi-$ resolution, obtainable from (3.1). In particular, $\pi_{s} M$ has the following finite, minimal, $\mathbb{Z} \pi$-presentation

$$
\pi_{s} M=\text { coker }\left(d_{s+2}: \mathbb{Z} \pi \otimes C_{s+2} \rightarrow \mathbb{Z} \pi \otimes C_{s+1}\right) .
$$

Taking $\pi$-coinvariants in equation (4.3) (that is, passing from the group $\pi_{s} M$ to its coinvariants $\left(\pi_{s} M\right)_{\pi}:=\pi_{s} M / I \pi \cdot \pi_{s} M$, where $I \pi \subset \mathbb{Z} \pi$ is the augmentation ideal), we find out from minimality that

$$
\left(\pi_{s} M\right)_{\pi}=C_{s+1}
$$

a finitely generated free abelian group, with combinatorially determined rank.

Guided by this result, we are going to look in the sequel at $\pi_{s} E$ as $\mathbb{Z} N$-module, more precisely at the $N$-coinvariants,

$$
\left(\pi_{s} E \otimes \mathbb{K}\right)_{N}:=\left(\pi_{s} E \otimes \mathbb{K}\right) /(I N \otimes \mathbb{K}) \cdot\left(\pi_{s} E \otimes \mathbb{K}\right)
$$

with arbitrary field coefficients. Note that $N$ is a normal subgroup of $\pi$, and the $\mathbb{Z} N$-module structure on $\pi_{s} E$ comes from the $\mathbb{Z} \pi$-module structure of $\pi_{s} M$, by restriction of scalars. Consequently, $\left(\pi_{s} E \otimes \mathbb{K}\right)_{N}$ has a naturally induced $\mathbb{K} \mathbb{Z}$-module structure.

We will completely describe this structure, again solely in terms of $\mathbf{m}$ and $s$. Here is our second main result in this section.

Theorem 4.4 Let $E(\mathbf{m}, s), 2 \leq s<r$, be the total space of the Alexander cover of $M(\mathbf{m}, s)$, with respect to the canonical 1-marking of $\pi_{1}(M(\mathbf{m}, s))=$ $\mathbb{F}(\mathbf{m})$. Set $N(\mathbf{m})=\pi_{1}(E(\mathbf{m}, s))$. Let $\mathbb{K}$ be an arbitrary field. Then the $\mathbb{K} \mathbb{Z}$-module structure of the $N(\mathbf{m})$-coinvariants of the first higher non-trivial homotopy group of $E(\mathbf{m}, s)$ is given by

$$
\left(\pi_{s} E(\mathbf{m}, s) \otimes \mathbb{K}\right)_{N(\mathbf{m})}=\left\{\begin{array}{cl}
(\mathbb{K} \mathbb{Z})^{z_{s}} \oplus \mathbb{K}^{z_{s+1}}, & \text { for } s<r-1 ; \\
(\mathbb{K} \mathbb{Z})^{\sigma_{r}}, & \text { for } s=r-1,
\end{array}\right.
$$

where $\left\{\sigma_{j}:=\sigma_{j}(\mathbf{m})\right\}_{0 \leq j \leq r}$ are the elementary symmetric functions evaluated at $\mathbf{m}$, and $\left\{z_{j}\right\}_{0 \leq j \leq r}$ are defined by (3.14). 
Proof Firstly, we infer from (4.3) that

$$
\left(\pi_{s} E \otimes \mathbb{K}\right)_{N}=\text { coker }\left(\mathbb{K} \mathbb{Z} \otimes_{\mathbb{Z} \pi} d_{s+2}: \mathbb{K} \mathbb{Z} \otimes C_{s+2} \rightarrow \mathbb{K} \mathbb{Z} \otimes C_{s+1}\right),
$$

as $\mathbb{K} \mathbb{Z}$-modules.

Once again, we may replace $\mathbb{K} \mathbb{Z} \otimes_{\mathbb{Z} \pi} C_{\bullet}(\tilde{Y})$ by $\mathbb{K} \mathbb{Z} \otimes_{\mathbb{Z} \pi} C_{\bullet}(\pi)$, as explained in $\$ 3.1$ and 33.5 , to arrive at

$$
\left(\pi_{s} E \otimes \mathbb{K}\right)_{N}=\text { coker }\left((\tau-1) \otimes \partial_{s+2}^{\prime}: \mathbb{K} \mathbb{Z} \otimes C_{s+2} \rightarrow \mathbb{K} \mathbb{Z} \otimes C_{s+1}\right),
$$

over $\mathbb{K} \mathbb{Z}$

All assertions to be proved follow now from (4.6) above, by using Lemma 3.6 and the $\mathbb{K} \mathbb{Z}$ analog of (3.13).

Remark 4.5 Upon applying Remark 3.8 to the above theorem, we see that $\pi_{s} E(\mathbf{m}, s)$ is not finitely generated over the group ring $\mathbb{Z} \pi_{1}(E(\mathbf{m}, s))$, if $s<r$. This is in striking contrast with the strong finiteness property of $\pi_{s} M(\mathbf{m}, s)=$ $\pi_{s} E(\mathbf{m}, s)$ over $\mathbb{Z} \pi_{1}(M(\mathbf{m}, s))$, recalled at the beginning of 4.3 . The nonfiniteness of $\pi_{r-1} E(\mathbf{m}, r-1)$ over $\mathbb{Z} N(\mathbf{m})$, in the case when $m_{i}>1$, for all $i$, is related to a certain non-finiteness homological property of the BestvinaBrady groups $N(\mathbf{m})$, see [1], as first noticed by Stallings [38.

Acknowledgements We are grateful to Alex Suciu for numerous, very useful discussions. This work started while the third author was visiting the University of Bordeaux, and it was finished during his stay at the Mathematical Sciences Research Institute, Berkeley. He is thankful to both institutions, for support and excellent working facilities.

A.D.R. Choudary and A. Dimca have been partially funded for this research by a grant from Higher Education Commission, Pakistan. S. Papadima has been partially supported by CERES grant 4-147/12.11.2004 of the Romanian Ministry of Education and Research. 


\section{References}

[1] M. Bestvina and N. Brady: Morse theory and finiteness properties of groups, Invent. Math. 129 (1997), 445-470. MathReview

[2] D. Cohen, G. Denham and A. Suciu: Torsion in Milnor fiber homology, Algebraic and Geometric Topology 3 (2003), 511-535. MathReview

[3] D. Cohen, A. Dimca and P. Orlik: Nonresonance conditions for arrangements, Annales de l'Insitut Fourier 53 (2003), 1883-1896. MathReview

[4] D. Cohen and A. Suciu: On Milnor fibrations of arrangements, J. London Math. Soc. 51 (1995), 105-119. MathReview

[5] P. Deligne: Le groupe fondamental du complément d'une courbe plane n'ayant que des points doubles ordinaires est abélien, Sém. Bourbaki 1979/80, LNM 842, pp.1-10, Springer-Verlag, Berlin 1981. MathReview

[6] A. Dimca: Singularities and Topology of Hypersurfaces, Universitext, SpringerVerlag, 1992. MathReview

[7] A. Dimca: Sheaves in Topology, Universitext, Springer-Verlag, 2004. MathReview

[8] A. Dimca: Hyperplane arrangements, $M$-tame polynomials and twisted cohomology, Commutative Algebra, Singularities and Computer Algebra, Eds. J. Herzog, V. Vuletescu, NATO Science Series, Vol. 115, Kluwer (2003), 113-126. MathReview

[9] A. Dimca and A. Némethi: Hypersurface complements, Alexander modules and monodromy, Proceedings of the 7th Workshop on Real and Complex Singularities, Sao Carlos, 2002, M. Ruas and T. Gaffney Eds., Contemp. Math. AMS 354 (2004), 19-43. MathReview

[10] A. Dimca and S. Papadima: Hypersurface complements, Milnor fibers and higher homotopy groups of arrangements, Annals of Math. 158 (2003), 473507. MathReview

[11] A. Dimca and S. Papadima: Equivariant chain complexes, twisted homology and relative minimality of arrangements, Ann. Scient. Ec. Norm. Sup. 37 (2004), 449-467. MathReview

[12] M. Falk: Homotopy types of line arrangements, Invent. Math. 111 (1993), 139150. MathReview

[13] K.-M. Fan: Direct product of free groups as the fundamental group of the complement of a union of lines, Michigan Math. J. 44 (1997), 283-291. MathReview

[14] W. Fulton: On the fundamental group of the complement of a nodal curve, Annals of Math. 111 (1980), 407-409. MathReview

[15] A. Hattori: Topology of $\mathbb{C}^{n}$ minus a finite number of affine hyperplanes in general position, J. Fac. Sci. Univ. Tokyo 22 (1975), 205-219. MathReview 
[16] J. A. Hillman: Alexander Ideals of Links, LNM 895, Springer-Verlag, 1981. MathReview

[17] P. J. Hilton and U. Stammbach: A Course in Homological Algebra, SpringerVerlag, 1971. MathReview

[18] M. Jambu and S. Papadima: A generalization of fiber-type arrangements and a new deformation method, Topology 37 (1998), 1135-1164. MathReview

[19] T. Jiang and S.S.-T. Yau: Topological invariance of intersection lattices of arrangements in $\mathbb{C P}^{2}$, Bull. Amer. Math. Soc. 29 (1993), 88-93. MathReview

[20] T. Jiang and S.S.-T. Yau: Diffeomorphic types of the complements of arrangements of hyperplanes, Compositio Math. 92 (1994), 133-155. MathReview

[21] Y. Kawahara: The mixed Hodge structure on the fundamental group of a complement of hyperplanes, Topology and its Applications 118 (2002), 131-145. MathReview

[22] Libgober, A.: Alexander modules of plane algebraic curves. Contemporary Mathematics, 20 (1983), 231-247 MathReview

[23] A. Libgober: Alexander invariants of plane algebraic curves, Proc. Symp. Pure Math. 40, Part 2, AMS (1983), 135-144. MathReview

[24] A. Libgober: Homotopy groups of the complements to singular hypersurfaces, II, Annals of Math. 139 (1994), 117-144. MathReview

[25] Libgober, A.: The topology of complements to hypersurfaces and nonvanishing of a twisted de Rham cohomology, AMS/IP Studies in Advanced Math. 5 (1997), 116-130 MathReview

[26] Libgober, A.: Eigenvalues for the monodromy of the Milnor fibers of arrangements. In: Libgober, A., Tibăr, M. (eds) Trends in Mathematics: Trends in Singularities. Birkhäuser, Basel (2002), 141-150 MathReview

[27] J. Milnor: Singular Points of Complex Hypersurfaces, Annals of Math. Studies 61, Princeton Univ. Press, 1968. MathReview

[28] M. Oka and K. Sakamoto: Product theorem of the fundamental group of a reducible curve, J. Math. Soc. Japan 30 (1978), 599-602. MathReview

[29] P. Orlik and L. Solomon: Combinatorics and topology of complements of hyperplanes, Invent. Math. 56 (1980), 167-189. MathReview

[30] P. Orlik and H. Terao: Arrangements of Hyperplanes, Grundlehren 300, Springer-Verlag, 1992. MathReview

[31] P. Orlik and H. Terao: Arrangements and Hypergeometric Integrals, Math. Soc. of Japan Memoirs 9, Tokyo, 2001. MathReview

[32] S. Papadima: Generalized $\bar{\mu}$-invariants for links and hyperplane arrangements, Proc. London Math. Soc. 84 (2002), 492-512. MathReview

[33] S. Papadima and A. Suciu: Higher homotopy groups of complements of complex hyperplane arrangements, Advances in Math. 165 (2002), 71-100. MathReview 
Some analogs of Zariski's Theorem on nodal line arrangements

[34] R. Randell: Lattice-isotopic arrangements are topologically isomorphic, Proc. Amer. Math. Soc. 107 (1989), 555-559. MathReview

[35] R. Randell: Homotopy and group cohomology of arrangements, Topology Appl. 78 (1997), 201-213. MathReview

[36] G. Rybnikov: On the fundamental group of the complement of a complex hyperplane arrangement, DIMACS Tech. Report 94-13 (1994), 33-50; available at arXiv:math.AG/9805056

[37] D. Siersma: The vanishing topology of non isolated singularities, in: D. Siersma et al.(eds) New Developments in Singularity Theory, Kluwer (2001), pp. 447472. MathReview

[38] J. R. Stallings: A finitely presented group whose 3-dimensional integral homology is not finitely generated, Am. J. Math. 85 (1963), 541-543. MathReview

[39] G. W. Whitehead: Elements of Homotopy Theory, Grad. Texts in Math. 61, Springer-Verlag, 1978. MathReview

[40] O. Zariski: On the problem of existence of algebraic functions of two variables possessing a given branch curve, Amer. J. Math. 51 (1929), 305-328.

Department of Mathematics, Central Washington University

Ellensburg, Washington 98926, USA and

School of Mathematical Sciences, GC University Lahore, Pakistan

Laboratoire J.A. Dieudonné, UMR du CNRS 6621

Université de Nice-Sophia-Antipolis, Parc Valrose

06108 Nice Cedex 02, France

and

Inst. of Math. "Simion Stoilow", P.O. Box 1-764, RO-014700 Bucharest, Romania

Email: choudary@cwu.edu, dimca@math.unice.fr, Stefan.Papadima@imar.ro

Received: 18 October 2004 Revised: 12 May 2005 